\title{
Hipoacusia: Tamizaje, Rehabilitar, Comunicar
}

\section{Hearing loss: Screen, Rehabilitate, Communicate}

Sofía Waissbluth A. ${ }^{1}$

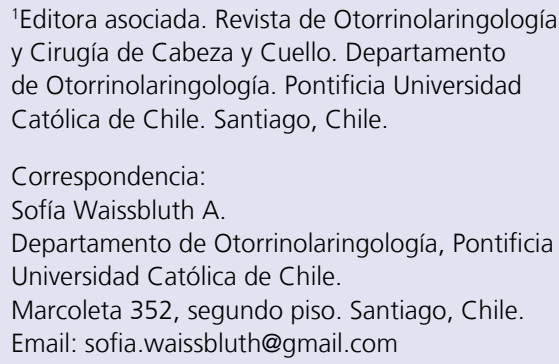

El día 3 de marzo es el Día Internacional de la Audición, día establecido por la Organización Mundial de la Salud (OMS) en el año 2007. La ideología se basa en compartir información, promover acciones para prevenir la pérdida de audición y mejorar las intervenciones para los pacientes que presentan hipoacusia. Previo al año 2016, era conocido como "Día Internacional del Cuidado del Oído"1. Se sabe que alrededor de 466 millones de personas a nivel mundial presentan una pérdida de audición discapacitante, y de ellos, 34 millones son niños. Esta cifra alcanzaría los 900 millones en el año 2050. Además, el 60\% de las hipoacusias en la infancia se deben a causas prevenibles ${ }^{2}$.

Todos los años, la OMS elige un tema específico para concientizar a la población sobre la hipoacusia. Temas previamente elegidos han sido: "Escucha de por vida", "Escucha el futuro", "Actuar contra la pérdida de audición: una buena inversión", "Pérdida de audición en la niñez", "Escuchar sin riesgos", "El cuidado del oído puede evitar la pérdida auditiva", y "Audición sana, vida feliz - Salud auditiva del adulto mayor" 3 . La fecha 3 de marzo fue elegida por la similitud de las cifras " 3 - 3" con la forma de nuestros oídos. Este día ofrece una oportunidad única para atraer la atención de los medios de comunicación, responsables políticos, administradores, profesionales de la salud y el público en general hacia la causa de la pérdida de audición ${ }^{1}$. Este año, el tema es: "Hearing Care for ALL! Screen, Rehabilitate, Communicate" y marcará el lanzamiento del primer informe mundial sobre la audición. La idea es lograr un flujo continuo de actividad promocional durante las 24 horas que dura el Día Internacional de la Audición ${ }^{4}$. Una forma de contribuir es con el uso de los medios de comunicación social, colocando \#hearathon2021, \#worldhearingday, \#hearingcare o \#safelistening y \#hearingcare para Facebook o Twitter. Tienen, además, un grupo en el servicio de red social Facebook al cual uno se puede unir: The World Hearing Forum Changemakers.

Generar conciencia del problema, fomentar la educación, desarrollar estrategias terapéuticas e implementar intervenciones tempranas son algunas de las tareas que tenemos como profesionales de la salud. La principal herramienta que tenemos hoy en día para implementar a los pacientes hipoacúsicos son los audífonos. Se ha descrito que aproximadamente un tercio de las personas mayores de 65 años a nivel mundial presentan una hipoacusia discapacitante ${ }^{2}$. En Chile, se crea en el año 2007 el sistema de Garantías Explícitas en Salud de la hipoacusia en el mayor de 65 años, con la rehabilitación de la comunicación mediante el uso de audífonos ${ }^{5}$. Uno de los principales problemas que se han visto en este grupo es la adherencia al uso de los audífonos, que se estima varía entre $40 \%$ y $80 \%{ }^{6-8}$.

En este número de la revista, se presenta un ensayo clínico aleatorizado controlado por Cardemil y colaboradores que demuestra que un programa de rehabilitación auditiva basada en Active Communication Education (ACE) mejora la adherencia al uso del audífono en pacientes adultos mayores con hipoacusia. Demuestran que la adherencia aumenta significativamente con el uso de un programa de rehabilitación auditiva, y que este efecto se mantiene hasta al menos un año después de haber iniciado la intervención. Se ocupó un programa basado en solución de problemas y 
monitorización telefónica, con un seguimiento de los pacientes que se realizó cada 3 meses. Este estudio nos muestra que la implementación con audífonos es compleja, y requiere de un seguimiento cercano y basado en las necesidades de los pacientes.

Pero no todo está en relación a niveles de audición sino también en la capacidad de entender lo que dicen las personas. Existe un grupo de pacientes que presentan un trastorno del procesamiento auditivo (TPA), siendo este un desafío para los otorrinolaringólogos. En este número de la revista, Marcotti y Rivera resumen los hallazgos de la evaluación electrofisiológica del procesamiento auditivo e incluyen a las respuestas auditivas complejas de tronco cerebral o cABR, los potenciales evocados auditivos de latencia media y tardía, el potencial de disparidad o MMN y el componente P300. Reportan que existen diferencias entre personas normoyentes y pacientes con TPA, pero que existe una gran variabilidad entre los pacientes por lo que difícilmente se puede establecer un perfil con estas pruebas electrofisiológicas. Otra limitante mencionada por los autores es la ausencia de valores normativos a nivel nacional.

La investigación en relación a la audición y sus alteraciones avanza a pasos agigantados, con lo cual estamos entendiendo las relaciones entre cognición y audición, la fisiología, bases genéticas y mecanismos moleculares del desarrollo de la cóclea y de la vía auditiva, y de la hipoacusia. Cada día se publican artículos de muy alto nivel que amplían nuestros conocimientos. Como profesionales de la salud, somos una de las fuentes primordiales de información médica para los pacientes. De- bemos ocupar estrategias, como las sugeridas por la OMS durante el Día Internacional de la Audición, para generar conciencia sobre la importancia de la audición y del impacto de la hipoacusia.

\section{Bibliografía}

1. Chadha S. International Ear Care Day-3rd March. J Laryngol Otol. 2014;128:221-222. doi: 10.1017/ S0022215114000413.

2. WHO. Deafness and hearing loss. Disponible en: https://www.who.int/news-room/fact-sheets/detail/ deafness-and-hearing-loss\#: :text=Hearing $\% 20$ loss $\% 20$ and $\% 20$ deafness,moderate $\% 2 \mathrm{C} \% 20$ severe\%2C\%20or\%20profound. Consultado: 1 de febrero de 2021.

3. WHO. World Hearing Day. Disponible en: https:// www.who.int/campaigns/world-hearing-day. Consultado el: 1 de febrero de 2021.

4. WHO. World Hearing Day 2021. Disponible en: https://www.who.int/campaigns/world-hearingday/2021. Consultado: 1 de febrero de 2021.

5. MINSAL. Guía Clínica Hipoacusia bilateral en personas de 65 años y más que requieren uso de audífono. Disponible en: http://www.redsalud.gov.cl/ archivos/guiasges/hipoacusia.pdf. Consultado: $1 \mathrm{de}$ febrero de 2021.

6. Pérez S, del Villar J, Rahal M. Cisternas A. Evaluación programa de audífonos para la tercera edad. Rev Otorrinolaringol Cir Cabeza Cuello. 2003;63:189-191.

7. Tamblay N, Villalobos I, Pastene A, Rahal M. Impacto social del uso de audífonos en adultos mayores. Rev Otorrinolaringol Cir Cabeza Cuello. 2008;68:21-26. doi: 10.4067/S0718-48162008000100004.

8. León A, Ediap R, Carvallo R. Adherencia al uso de audífonos en adultos mayores del Servicio de Salud Aconcagua. Rev Otorrinolaringol Cir Cabeza Cuello. 2010;70:37-42. doi: 10.4067/S071848162010000100006. 